\title{
EXISTENCE OF PULLBACK ATTRACTORS FOR PULLBACK ASYMPTOTICALLY COMPACT PROCESSES
}

\author{
TOMÁS CARABALLO ${ }^{1}$, ALEXANDRE N. CARVALHO $^{2}$, JOSÉ A. LANGA $^{3}$, AND FELIPE RIVERO $^{4}$
}

\begin{abstract}
This paper is concerned with the existence of pullback attractors for evolution processes. Our aim is to provide results that extend the following results for autonomous evolution processes (semigroups)

i) An autonomous evolution process which is bounded dissipative and asymptotically compact has a global attractor.

ii) An autonomous evolution process which is bounded, point dissipative and asymptotically compact has a global attractor.

The extension of such results requires the introduction of new concepts and brings up some important differences between the asymptotic properties of autonomous and nonautonomous evolution processes. An application is considered to damped wave problem with non-autonomous damping.
\end{abstract}

\section{INTRODUCTION}

One of the central problems in dynamical systems is the study of the asymptotic behavior of evolution processes associated to the modeling of real world phenomena. When the model under study is an autonomous differential equation, the study of its asymptotic behavior is rather well established and many references on the subject are available (cf. Temam [17], Hale [8], Ladyzhenskaya [10], Babin-Vishik [1], Robinson [13] for example). However, if the evolution process comes from a non-autonomous differential equation, even though some nice references are already available (cf. Cheban [4], Chepyzhov-Vishik [6], Kloeden [9], Sell-You [15]), much is yet to be done.

The subject of this paper is the existence of pullback attractors for evolution processes. As we will see, even this fundamental question is not as well developed as its autonomous counterpart. Our aim is to give results that allow us to conclude the existence of pullback

\footnotetext{
${ }^{1}$ Partially supported by Ministerio de Ciencia e Innovación grant \# MTM2008-0088 and Junta de Andalucía grants \# P07-FQM-02468, \# FQM314, Spain.

${ }^{2}$ Partially supported by CNPq 305447/2005-0 and 451761/2008-1, CAPES/DGU 267/2008 and FAPESP 2008/53094-4, Brazil.

${ }^{3}$ Partially supported by Ministerio de Ciencia e Innovación grants \# MTM2008-0088, \# PBH2006-0003PC, and Junta de Andalucía grants \# P07-FQM-02468, \# FQM314, Spain.

${ }^{4}$ Partially supported by Ministerio de Ciencia e Innovación grant \# MTM2008-0088 and Junta de Andalucía grants \# P07-FQM-02468, \# FQM314, Spain.
} 
attractors for pullback asymptotically compact and pullback point (or bounded) dissipative processes. We also point out the features of non-autonomous evolution processes must be considered to obtain results which are analogues to those for the autonomous evolution processes emphasizing the distinction between the autonomous and non-autonomous cases.

To better explain the results in the paper we introduce some basic terminology. Let $X$ be a metric space and $\mathrm{d}(\cdot, \cdot): X \times X \rightarrow[0, \infty)$ be its metric. Denote by $\mathcal{C}(X)$ the set of all continuous transformations from $X$ into itself. An evolution process in $X$ is a family of maps $\{S(t, s): t \geqslant s\} \subset \mathcal{C}(X)$ with the following properties

1) $S(t, t)=I$, for all $t \in \mathbb{R}$,

2) $S(t, s)=S(t, \tau) S(\tau, s)$, for all $t \geqslant \tau \geqslant s$,

3) $\left\{(t, s) \in \mathbb{R}^{2}: t \geqslant s\right\} \times X \ni(t, s, x) \mapsto S(t, s) x \in X$ is continuous.

The processes $\{S(t, s): t \geqslant s\}$ for which $S(t, s)=S(t-s, 0)$ for all $t \geqslant s$ are called autonomous evolution process and the family $\{T(t): t \geqslant 0\}$ given by $T(t)=S(t, 0)$, $t \geqslant 0$ is called a semigroup; that is, a family $\{T(t): t \geq 0\}$ which satisfies

1) $T(0)=I$

2) $T(t+s)=T(t) T(s)$, for all $t, s \geqslant 0$,

3) $[0, \infty) \times X \ni(t, x) \mapsto T(t) x \in X$ is continuous.

Reciprocally, given a semigroup $\{T(t): t \geqslant 0\}$ the family $\{T(t-s): t \geqslant s\}$ is an evolution process.

For an autonomous evolution process $\{S(t, s)=S(t-s, 0): t \geqslant s\}$, the behavior of solutions as $t \rightarrow \infty$, which is called the forwards dynamics, is the same as the behavior of solutions as $s \rightarrow-\infty$, which is called the pullback dynamics. For general processes, these two dynamical limits are totally unrelated and can produce entirely different qualitative properties (see [3]).

The pullback orbit of a subset $B$ of $X$ at time $t \in \mathbb{R}$, is defined by $\gamma_{p}(B, t):=\bigcup_{s \leqslant t} S(t, s) B$, where $S(t, s) B:=\{S(t, s) x: x \in B\} .\{S(t, s): t \geqslant s\}$ is said pullback bounded if $\gamma_{p}(B, t)$ is bounded whenever $B$ is a bounded subset of $X$ and for each $t \in \mathbb{R}$. A set $K(t) \subset X$ pullback attracts a set $C$ at time $t$ under $\{S(t, s): t \geqslant s\}$ if

$$
\lim _{s \rightarrow-\infty} \operatorname{dist}(S(t, s) C, K(t))=0 .
$$

A family $\{K(t): t \in \mathbb{R}\}$ pullback attracts bounded subsets of $X$ under $\{S(t, s): t \geqslant s\}$ if $K(t)$ pullback attracts all bounded subsets at $t$ under $\{S(t, s): t \geqslant s\}$, for each $t \in \mathbb{R}$. Given $t \in \mathbb{R}, B(t) \subset X$ pullback absorbs bounded subsets of $X$ at time $t$ if, for each bounded subset $B$ of $X$, there exists $T=T(t, B) \leqslant t$ such that

$$
S(t, s) B \subset B(t) \text {, for all } s \leqslant T(t, B) .
$$


A family $\{B(t): t \in \mathbb{R}\}$ pullback absorbs bounded subsets of $X$ if $B(t)$ pullback absorbs bounded sets at time $t$, for each $t \in \mathbb{R}$. If there exists a family $\{B(t): t \in \mathbb{R}\}$ of bounded sets which pullback absorbs bounded subsets of $X$ then we say that the evolution process $\{S(t, s): t \geqslant s\}$ is pullback bounded dissipative. In a completely similar way we define the notions of pullback point dissipative or pullback compact dissipative processes. Let $\{B(t)$ : $t \in \mathbb{R}\}$ a family of subsets of $X$. We say that this family is invariant with respect to the process $\{S(t, s): t \geqslant s\}$ if

$$
S(t, s) B(s)=B(t) \text {, for all } t \geqslant s .
$$

Definition 1.1. A family $\{\mathcal{A}(t): t \in \mathbb{R}\}$ of compact subsets of $X$ is said a pullback attractor for the evolution process $\{S(t, s): t \geqslant s\}$ if it is invariant, pullback attracts bounded subsets of $X$ and is minimal; that is, if there is another invariant family $\{C(t): t \in$ $\mathbb{R}\}$ which pullback attracts bounded subsets of $X$, then $A(t) \subseteq C(t)$, for all $t \in \mathbb{R}$.

An autonomous evolution process $\{S(t-s): t \geq s\}$ has a pullback attractor $\{\mathcal{A}(t): t \in \mathbb{R}\}$ if and only if the semigroup $\{S(t): t \geq 0\}$ has a global attractor $\mathcal{A}$ (in the sense of [8]) and in either case $\mathcal{A}(t)=\mathcal{A}$ for all $t \in \mathbb{R}$.

We remark that the minimality requirement in Definition 1.1 is additional relative to the theory of attractors for semigroups. This minimality requirement is essential to ensure uniqueness of pullback attractors. Its inclusion is related to the weakening of the invariance property imposed by the non-autonomous nature of general evolution processes. If $\{S(t)$ : $t \geqslant 0\}$ is a semigroup and $\{S(t-s): t \geqslant s\}$ is the process associated to it, there may exist a family $\{\mathcal{A}(t): t \in \mathbb{R}\}$ of compact invariant sets that pullback attracts bounded subsets and is not minimal. Indeed, if $S(t-s)=e^{-(t-s)} x_{0}, x_{0} \in \mathbb{R}, t \geqslant s$ and $c \geqslant 0$ the family $\left\{\left[-c e^{-t}, c e^{-t}\right]: t \in \mathbb{R}\right\}$ is invariant, $\left[-c e^{-t}, c e^{-t}\right]$ is compact and attracts bounded subsets of $\mathbb{R}$ at time $t$ for each $t \in \mathbb{R}$.

Remark 1.2. Recall that, a global solution for an evolution process $\{S(t, s): t \geqslant s\}$ is a function $\xi: \mathbb{R} \rightarrow X$ such that $S(t, s) \xi(s)=\xi(t)$ for all $t \geq s$. We say that a global solution $\xi: \mathbb{R} \rightarrow X$ of an evolution process is backwards bounded if there is a $\tau \in \mathbb{R}$ such that $\{\xi(t): t \leqslant \tau\}$ is a bounded subset of $X$.

If an evolution process $\{S(t, s): t \geqslant s\}$ has a pullback attractor $\{\mathcal{A}(t): t \in \mathbb{R}\}$ and $\xi: \mathbb{R} \rightarrow X$ is a backwards bounded solution, then $\xi(t) \in \mathcal{A}(t)$ for all $t \in \mathbb{R}$. If we require, in addition, that the pullback attractor $\{A(t): t \in \mathbb{R}\}$ has the property that $\cup_{s \leq \tau} A(s)$ is bounded for each $\tau \in \mathbb{R}$, the requirement that the pullback attractor is minimal in Definition 1.1 can be dropped and

$$
A(t)=\{\xi(t): \xi: \mathbb{R} \rightarrow X \text { is a global backwards bounded solution of }\{S(t, s): t \geqslant s\}\}
$$


The notion of a pullback asymptotically compact evolution process is naturally associated with evolution processes which posses a pullback attractor.

Definition 1.3. An evolution process $\{S(t, s): t \geqslant s\}$ in a metric space $X$ is said pullback asymptotically compact if, for each $t \in \mathbb{R}$, sequence $\left\{s_{k}\right\}_{k \in \mathbb{N}}$ in $(-\infty, t]$ and bounded sequence $\left\{x_{k}\right\}_{k \in \mathbb{N}}$ in $X$ such that

- $s_{k} \stackrel{k \rightarrow \infty}{\longrightarrow}-\infty$ and

- $\left\{S\left(t, s_{k}\right) x_{k}: k \in \mathbb{N}\right\}$ is bounded,

the sequence $\left\{S\left(t, s_{k}\right) x_{k}\right\}_{k \in \mathbb{N}}$ has a convergent subsequence.

Next we give two characterizations of autonomous evolution processes which posses global attractors. Both characterizations are widely used to obtain existence of pullback attractors for autonomous evolution processes. We will first formulate them in the classical way, and later we will rewrite them taking into account the framework described above. (See [8] or [10] for more details of the autonomous formulation).

Theorem 1.4. If $\{S(t): t \geq 0\}$ is a semigroup, the following conditions are equivalent:

i) $\{S(t): t \geq 0\}$ possesses a global attractor.

ii) $\{S(t): t \geq 0\}$ is bounded dissipative and asymptotically compact.

iii) $\{S(t): t \geq 0\}$ is bounded, point dissipative and asymptotically compact.

Condition $i$ ) gives the following characterization of autonomous processes which have pullback attractors

Theorem 1.5. If $\{S(t): t \geq 0\}$ is a semigroup, the autonomous evolution process $\{S(t-s)$ : $t \geq s\}$ has a pullback attractor if and only if it is pullback asymptotically compact and pullback bounded dissipative.

On the other hand, condition $i i i)$ gives we have that

Theorem 1.6. If $\{S(t): t \geq 0\}$ is a semigroup, the autonomous evolution process $\{S(t-s)$ : $t \geq s\}$ has a pullback attractor if and only if $\{S(t-s): t \geq 0\}$ is pullback bounded, pullback point dissipative and pullback asymptotically compact.

The decision about which characterization should be used in a given application depends on the properties of the model under study. The first result is widely used associated to energy estimates for asymptotically compact autonomous evolution processes and the second is mostly associated to asymptotically compact gradient autonomous evolution processes with a bounded set of equilibria.

Our aim is to provide extensions of the above results to the case of general (autonomous or not) evolution processes. As we will see in Section 2, even the statement of such extensions in the general case will require the introduction of several new (natural) concepts. 
The paper is organized as follows. In Section 2 we state the main results of the paper, introduce the necessary definitions and terminology to be able to state and apply them. In Section 3 we prove the analogues to Theorem 1.5 and prove a result that implies pullback asymptotic compactness. In Section 4 we prove the analogue of Theorem 1.6 .

\section{Statement of the Results AND their APPliCAtions}

Let us start recalling the definition of pullback $\omega$ - limit sets.

Definition 2.1. Let $\{S(t, s): t \geqslant s\}$ be an evolution process in a metric space $X$ and $B$ be a subset of $X$. The pullback $\omega$-limit of $B$ is defined by

$$
\omega(B, t):=\bigcap_{\sigma \leqslant t} \overline{\bigcup_{s \leqslant \sigma} S(t, s) B} .
$$

For each subset $B$ of $X$, it holds that

$$
\begin{array}{r}
\omega(B, t)=\left\{y \in X: \text { there are sequences }\left\{s_{k}\right\}_{k \in \mathbb{N}} \text { in }(-\infty, t], s_{k} \stackrel{k \rightarrow \infty}{\longrightarrow}-\infty\right. \\
\text { and } \left.\left\{x_{k}\right\}_{k \in \mathbb{N}} \text { in } B \text {, such that } y=\lim _{k \rightarrow \infty} S\left(t, s_{k}\right) x_{k}\right\} .
\end{array}
$$

The following theorem gives a characterization of evolution processes with a pullback attractor. So far, it is the only result available to obtain existence of pullback attractors in applications (see [8, 10, 12, 13, 17] for the autonomous case).

Theorem 2.2. Let $\{S(t, s): t \geqslant s\}$ be an evolution process in a metric space $X$. Then, the following statements are equivalent

- $\{S(t, s): t \geqslant s\}$ has a pullback attractor $\{\mathcal{A}(t): t \in \mathbb{R}\}$.

- There exists a family of compact sets $\{K(t): t \in \mathbb{R}\}$ that pullback attracts bounded subsets of $X$ under $\{S(t, s): t \geqslant s\}$.

In either case

$$
\mathcal{A}(t)=\overline{\bigcup\{\omega(B, t): B \subset X, B \text { bounded }\}}
$$

and $\{\mathcal{A}(t): t \in \mathbb{R}\}$ is minimal in the sense that, if there exists another family of closed bounded sets $\{\hat{\mathcal{A}}(t): t \in \mathbb{R}\}$ which pullback attracts bounded subsets of $X$ under $\{S(t, s)$ : $t \geqslant s\}$, then $\mathcal{A}(t) \subseteq \hat{\mathcal{A}}(t)$, for all $t \in \mathbb{R}$.

This result is proved in [2, 12] and we provide a short proof in Section 3 for completeness.

The application of such result for processes which are not compact may be difficult because one must find a compact set $K(t)$ which pullback attracts bounded subsets of $X$ for each $t \in \mathbb{R}$. We aim to provide a few alternative results to prove existence of pullback attractors in order to have a theory as complete as in the autonomous case.

We prove in Section 3 that 
Theorem 2.3. If $\{S(t, s): t \geqslant s\}$ is pullback bounded dissipative and pullback asymptotically compact, then $\mathcal{A}(t)$ given by $(2.2)$ is closed, invariant, pullback attracts bounded subsets of $X$ at time $t$, and the family $\{\mathcal{A}(t): t \in \mathbb{R}\}$ is minimal among the families $\{B(t): t \in \mathbb{R}\}$ such that $B(t)$ is closed and pullback attracts bounded subsets of $X$ at time $t$.

Observe that the previous result does not give any compactness of $\mathcal{A}(t)$. This is not a restriction in the finite dimensional case, as $\mathcal{A}(t)$ is actually bounded and closed. However, the result shows a first difference with respect to analogous results for autonomous evolution processes in the infinite dimensional case. Getting the same kind of results requires to adopt different strategies, any of them imposing new hypotheses on the dynamics of the processes.

Definition 2.4. We say that an evolution process $\{S(t, s): t \geqslant s\}$ is pullback strongly bounded dissipative if, for each $t \in \mathbb{R}$, there is a bounded subset $B(t)$ of $X$ which pullback attracts bounded subsets of $X$ at time $\tau$ for each $\tau \leqslant t$; that is, given a bounded subset $B$ of $X$ and $\tau \leqslant t$, there exists $s_{0}(\tau, D)$ such that $S(\tau, s) B \subset B(t)$, for all $s \leqslant s_{0}(\tau, D)$.

Note that the family $\{B(t): t \in \mathbb{R}\}$ given in the above definition does not need to have a bounded union. Nonetheless, we may choose it in such a way that, for each $t \in \mathbb{R}, \bigcup_{s \leqslant t} B(s)$ is bounded. The following theorem, proved in Section 3, gives a sufficient condition for the existence of a compact pullback attractor.

Theorem 2.5. If an evolution process $\{S(t, s): t \geqslant s\}$ is pullback strongly bounded dissipative and pullback asymptotically compact, then $\{S(t, s): t \geqslant s\}$ has a pullback attractor $\{\mathcal{A}(t): t \in \mathbb{R}\}$ with the property that $\bigcup_{s \leqslant t} \mathcal{A}(s)$ is bounded for each $t \in \mathbb{R}$.

To prove (in applications) that a process is asymptotically compact, we will need to assume that the evolution process is pullback strongly bounded as defined next

Definition 2.6. We say that an evolution process $\{S(t, s): t \geqslant s\}$ in $X$ is pullback strongly bounded if, for each $t \in \mathbb{R}$ and bounded subset $B$ of $X, \bigcup_{s \leqslant t} \gamma_{p}(B, s)$ is bounded.

Remark 2.7. If $\{S(t): t \geqslant 0\}$ is a semigroup, $\{S(t-s): t \geqslant s\}$ is pullback strongly bounded if and only if $\{S(t-s): t \geqslant s\}$ is pullback bounded if and only if $\{S(t): t \geqslant 0\}$ is a bounded semigroup.

For evolution processes which are pullback strongly bounded, the following result (proved in Section 3) gives sufficient conditions for pullback asymptotic compactness

Theorem 2.8. Let $\{S(t, s): s \leqslant t\}$ be a pullback strongly bounded process such that $S(t, s)=$ $T(t, s)+U(t, s)$, where $U(t, s)$ is compact and there exits a non-increasing function

$$
k: \mathbb{R}^{+} \times \mathbb{R}^{+} \longrightarrow \mathbb{R}
$$

with $k(\sigma, r) \rightarrow 0$ when $\sigma \rightarrow \infty$, and for all $s \leqslant t$ and $x \in X$ with $\|x\| \leqslant r,\|T(t, s) x\| \leqslant$ $k(t-s, r)$. Then, the process $\{S(t, s): s \leqslant t\}$ is pullback asymptotically compact. 
In Section 4 we pursue a result that would enable us to conclude the existence of pullback attractors without having to prove pullback strong bounded dissipativeness but rather pullback strong point dissipativeness (see Hale [8], Raugel [12]). To that end, the notion of pullback asymptotic compactness and pullback dissipativeness associated to the elapsed time presented next is needed.

Definition 2.9. We say that an evolution process $\{S(t, s): t \geqslant s\}$ is pullback strongly asymptotically compact if for each $t \in \mathbb{R}$, bounded sequence $\left\{x_{k}: k \in \mathbb{N}\right\}$ in $X$, sequences $\left\{s_{k}: k \in \mathbb{N}\right\},\left\{\tau_{k}: k \in \mathbb{N}\right\}$ with $t \geqslant \tau_{k} \geqslant s_{k}$ and $\tau_{k}-s_{k} \stackrel{k \rightarrow \infty}{\longrightarrow} \infty$, then $\left\{S\left(\tau_{k}, s_{k}\right) x_{k}: k \in \mathbb{N}\right\}$ is relatively compact. If $\{S(t): t \geqslant 0\}$ is a semigroup, $\{S(t-s): t \geqslant s\}$ is pullback strongly asymptotically compact if and only if $\{S(t-s): t \geqslant s\}$ is pullback asymptotically compact if and only if $\{S(t): t \geqslant 0\}$ is asymptotically compact in the sense of [10]. The process $\{S(t, s): s \leqslant t\}$ is called strongly compact if for each time $t$ and $B \subset X$ there exits $a T_{B} \leqslant 0$ and a compact set $K \subset X$ such that $U(\tau, s) B \subset K$ for all $s \leqslant \tau \leqslant t$ with $\tau-s \geqslant T_{B}$.

Definition 2.10. Let $\{S(t, s): t \geqslant s\}$ be an evolution process in a metric space $X$. We say that a bounded set $B(t)$ of $X$ pullback strongly absorbs points (compact subsets) of $X$ at time $t$ if, for each $x \in X$ (compact subset $K$ of $X)$, there exist $\sigma_{x}>0\left(\sigma_{K}>0\right)$ such that $S(\tau, s)(x) \in B(t)(S(\tau, s)(K) \subset B(t))$ for all $s \leqslant \tau \leqslant t$ with $\tau-s \geqslant \sigma_{x}\left(\tau-s \geqslant \sigma_{K}\right)$. We say that $\{S(t, s): t \geqslant s\}$ is pullback strongly point dissipative (compact dissipative) if, for each $t \in \mathbb{R}$, there is a bounded subset $B(t) \subset X$ which pullback strongly absorbs points (compact subsets) of $X$ at time $t$.

Remark 2.11. If a set $B(t)$ pullback strongly absorbs points (compact subsets/bounded subsets) of $X$ at $t$, then it pullback strongly absorbs points (compact subsets/bounded subsets) of $X$ at $\tau$ for all $\tau \leqslant t$. Also, if $\{S(t): t \geqslant 0\}$ is a semigroup then $\{S(t-s): t \geqslant s\}$ is pullback strongly point dissipative (compact dissipative) if and only if $\{S(t-s): t \geqslant s\}$ is pullback point dissipative (compact dissipative) if and only if $\{S(t): t \geqslant 0\}$ is point dissipative (compact dissipative) in the sense of [8].

With these concepts we can prove the following result on existence of pullback attractors.

Theorem 2.12. Let $\{S(t, s): t \geqslant s\}$ be an evolution process with the property that, for each $t \in \mathbb{R}$ and $\tau>0,\{S(s, s-\tau): s \leqslant t\}$, is equicontinuous at $x$ for each $x \in X$. If $\{S(t, s): t \geqslant s\}$ is pullback strongly point dissipative, pullback strongly bounded and pullback strongly asymptotically compact, then $\{S(t, s): t \geqslant s\}$ is pullback strongly bounded dissipative. Consequently, $\{S(t, s): t \geqslant s\}$ has a pullback attractor $\{\mathcal{A}(t): t \in \mathbb{R}\}$ with the property that $\bigcup_{s \leqslant t} \mathcal{A}(s)$ is bounded for each $t \in \mathbb{R}$.

In applications, to prove the pullback strong asymptotic compactness property we use the following result 
Theorem 2.13. Let $\{S(t, s): s \leqslant t\}$ be a pullback strongly bounded process such that $S(t, s)=T(t, s)+U(t, s)$, where $U(t, s)$ is strongly compact and there exits a non-increasing function

$$
k: \mathbb{R}^{+} \times \mathbb{R}^{+} \longrightarrow \mathbb{R}
$$

with $k(\sigma, r) \rightarrow 0$ when $\sigma \rightarrow \infty$, and for all $s \leqslant t$ and $x \in X$ with $\|x\| \leqslant r,\|T(t, s) x\| \leqslant$ $k(t-s, r)$. Then, the process $\{S(t, s): s \leqslant t\}$ is pullback strongly asymptotically compact.

2.1. A pullback strongly bounded dissipative wave equation. In this section we give an application of Theorem 2.5. Let us consider the following nonautonomous wave equation

$$
\left\{\begin{array}{l}
u_{t t}+\beta(t) u_{t}=\Delta u+f(u) \text { in } \Omega \\
u(x, t)=0 \text { in } \partial \Omega
\end{array}\right.
$$

where $\Omega \subset \mathbb{R}^{n}$ is a bounded smooth domain $\mathbb{R}^{n}$. For $f: \mathbb{R} \rightarrow \mathbb{R}$ we assume that:

$$
f \in C^{2}(\mathbb{R}),\left|f^{\prime}(s)\right| \leqslant c\left(1+|s|^{p-1}\right), \limsup _{|s| \rightarrow \infty} \frac{f(s)}{s} \leqslant 0,
$$

with $c>0$ and $p<\frac{n}{n-2}$

Assume that $\beta: \mathbb{R} \rightarrow \mathbb{R}$ is a bounded, globally Lipschitz function and that there are

$$
\beta_{0} \leqslant \beta(t) \leqslant \beta_{1} \text { for some } \beta_{0}, \beta_{1} \in(0, \infty) \text {. }
$$

We will prove that the nonautonomous process associated to (2.3) has a pullback attractor by applying Theorem 2.8. Let $X=H_{0}^{1}(\Omega) \times L^{2}(\Omega)$, for $u_{t}=v$ and $w=\left(\begin{array}{l}u \\ v\end{array}\right)$, we rewrite $(2.3)$ as

$$
w_{t}=C(t) w+F(w)
$$

where

$$
C(t)=\left(\begin{array}{cc}
0 & I \\
-A & -\beta(t) I
\end{array}\right) \text { and } F(w)=\left(\begin{array}{c}
0 \\
f(u)
\end{array}\right)
$$

$A=-\Delta$ with Dirichlet boundary condition.

For each initial value $w_{0} \in X$ and initial time $s \in \mathbb{R}$, system (2.6) possesses a unique solution which can be written as

$$
S(t, s) w_{0}=L(t, s) w_{0}+U(t, s) w_{0}
$$

where $L(t, s)$ is the solution operator for $w_{t}=C(t) w$, and

$$
U(t, s) w_{0}=\int_{s}^{t} L(t, \tau) F\left(S(\tau, s) w_{0}\right) d \tau
$$

Let us prove that $\{S(t, s): t \geqslant s\}$ is pullback asymptotically compact using Theorem 2.8. 
EXISTENCE OF PULLBACK ATTRACTORS FOR PULLBACK ASYMPTOTICALLY COMPACT PROCESSEM

Proposition 2.14. There are positive constants $K>0, \alpha>0$, such that:

$$
\|L(t, s)\| \leqslant K e^{-\alpha(t-s)}, t \geqslant s .
$$

Proof: Consider the following function

$$
V(\varphi, \phi)=\frac{1}{2}|\varphi|_{H_{0}^{1}(\Omega)}^{2}+2 b(\varphi, \phi)_{L^{2}(\Omega)}+\frac{1}{2}|\phi|_{L^{2}(\Omega)}^{2},(\varphi, \phi) \in X
$$

For suitably small $b_{0}>0$ and $b \leq b_{0}$ we have that

$$
\begin{aligned}
\frac{1}{4}\left[|\varphi|_{H_{0}^{1}(\Omega)}^{2}+|\phi|_{L^{2}(\Omega)}^{2}\right] & \leqslant \frac{1}{2}|\varphi|_{H_{0}^{1}(\Omega)}^{2}+2 b(\varphi, \phi)+\frac{1}{2}|\phi|_{L^{2}(\Omega)}^{2} \\
& \leqslant \frac{3}{4}\left[|\varphi|_{H_{0}^{1}(\Omega)}^{2}+|\phi|_{L^{2}(\Omega)}^{2}\right] .
\end{aligned}
$$

If $\left(u(t, s), u_{t}(t, s)\right)$ is a solution of

$$
\left\{\begin{aligned}
u_{t t}+\beta(t) u_{t}-\Delta u=0 & \text { in } \Omega \\
u(x, t)=0 & \text { in } \partial \Omega
\end{aligned}\right.
$$

then

$$
\frac{d}{d t} V\left(u, u_{t}\right)=\left(u, u_{t}\right)_{H_{0}^{1}(\Omega)}+2 b\left(u_{t}, u_{t}\right)_{L^{2}(\Omega)}+2 b\left(u, u_{t t}\right)_{L^{2}(\Omega)}+\left(u_{t}, u_{t t}\right)_{L^{2}(\Omega)} .
$$

Therefore,

$$
\begin{aligned}
& \frac{d}{d t} V\left(u(t, s), u_{t}(t, s)\right) \\
& \quad=-(\beta(t)-2 b)\left|u_{t}\right|_{L^{2}(\Omega)}^{2}-2 b|u|_{H_{0}^{1}(\Omega)}^{2}-2 \beta(t)\left(u, u_{t}\right)_{L^{2}(\Omega)} \\
& \quad \leqslant-\left(\beta_{0}-2 b-\frac{b \beta_{1}}{\varepsilon}\right)\left|u_{t}\right|_{L^{2}(\Omega)}^{2}+\left(b \beta_{1} \varepsilon-b \lambda_{1}\right)|u|_{L^{2}(\Omega)}^{2}-b|u|_{H_{0}^{1}(\Omega)}^{2},
\end{aligned}
$$

with $\varepsilon=\frac{\lambda_{1}}{\beta_{1}}$ and $b_{0}>0$ small enough. Then we have that, for all $0<b \leq b_{0}$,

$$
\frac{d}{d t} V\left(u(t, s), u_{t}(t, s)\right)=-\alpha V\left(u(t, s) u_{t}(t, s)\right) .
$$

Hence,

$$
V(L(t, s)(\varphi, \phi)) \leqslant V(\varphi, \phi) e^{-\alpha(t-s)}
$$

and, consequently, $\|L(t, s)(\varphi, \phi)\|_{X}^{2} \leqslant K e^{-\alpha(t-s)}\|(\varphi, \phi)\|$ proving the result.

Theorem 2.15. $\{S(t, s): t \geqslant s\}$ is pullback strongly bounded dissipative.

Proof: Consider the energy functionals

$$
\mathcal{V}(\varphi, \phi)=\frac{1}{2}|\varphi|_{H_{0}^{1}(\Omega)}^{2}+2 b(\varphi, \phi)+\frac{1}{2}|\phi|_{L^{2}(\Omega)}^{2}-\int_{\Omega} G(\varphi),
$$


where $G(s)=\int_{0}^{s} f(\theta) d \theta$. As in (2.11) we obtain

$$
\frac{d}{d t} \mathcal{V}\left(u(t, s), u_{t}(t, s)\right) \leqslant-\alpha \mathcal{V}\left(u, u_{t}\right)
$$

From this we have that,

$$
\|S(t, s)(\varphi, \phi)\|_{X}^{2} \leqslant C(B) e^{-\alpha(t-s)}+K,
$$

for some constant $K$ and the result is proved.

Theorem 2.16. $\{U(t, s): t \geq s\}$ is compact.

Proof: Let $B \subset X$ be bounded and $w_{0}=\left(\begin{array}{l}u_{0} \\ v_{0}\end{array}\right) \in B$. Let $t \mapsto S(t, s) w_{0}$ be the solution of (2.6) which at time $s$ is $w_{0}$, and consider

$$
U(t, s) w_{0}=\int_{s}^{t} T(t, \theta) F\left(S(\theta, s) w_{0}\right) d \theta .
$$

The compactness of $U$ follows easily from the fact that $f$ is bounded from $H_{0}^{1}(\Omega)$ into $W^{1, p}(\Omega)$ for some $2>p>\frac{2 n}{n+2}$ and consequently into compact subsets of $L^{2}(\Omega)$.

Hence, applying Theorem 2.8 and Theorem 2.5, we can conclude that system (2.6) has a pullback attractor in $X$.

2.2. A pullback strongly point dissipative wave equation. We consider the damped wave equation in (2.3) with all the assumptions over $f(s)$ and $\beta(t)$ as follow

$$
\beta(t)=\left\{\begin{array}{lll}
\beta_{0} & \text { if } \quad t \leqslant 0 \\
b(t) & \text { in } \quad t>0
\end{array}\right.
$$

where $b(t)$ is a bounded and globally Lipschitz function with $b(0)=\beta_{0}$.

We already know that the process $\{S(t, s): s \leqslant t\}$ associated to this problem is pullback strongly asymptotically compact and pullback strongly bounded. We want to verify that it is also pullback strongly point dissipative and equicontinuous.

When $\beta(t)$ is constant we have an autonomous equation, so the semigroup associated $S(t, s)=\widetilde{S}_{0}(t-s)$ is point dissipative (see for example [8] or [17]), i.e. if $\beta(t) \equiv \beta_{0}$, there exists a set $B_{0} \subset X$ such that for each $x \in X, \widetilde{S}_{0}(t) x \subset B_{0}$ for all $t>\sigma_{x}$. We now define the following bounded sets

$$
B(t)=\bigcup_{\tau \leqslant t} \gamma_{p}\left(B_{0}, \tau\right)
$$

We take a fixed $t \in \mathbb{R}, x \in X$ and consider $S(\tau, s) x$ with $s \leqslant \tau \leqslant t$. If $t \leqslant 0$ we know that there exits a $\sigma_{x}>0$ such that $S(\tau, s) x=\widetilde{S}_{0}(\tau-s) x \in B_{0} \subset B(t)$ for all $\tau-s \geqslant \sigma_{x}$. Otherwise if $t>0$ we can take a fixed $\widetilde{\tau}<0$ such that $s \leqslant \widetilde{\tau}<\tau$ and $S(\tau, s) x=S(\tau, \widetilde{\tau}) S(\widetilde{\tau}, s) x$. Therefore there exits a $\sigma_{x}>0$ such that $S(\widetilde{\tau}, s) x \in B_{0}$ for all $\tilde{\tau}-s \geqslant \sigma_{x}$. Therefore $S(\tau, s) x \subset B(t)$ for all $\tau-s \geqslant \widetilde{\tau}-s \geqslant \sigma_{x}$. So $\{S(t, s): s \leqslant t\}$ is pullback strongly point dissipative. 
Let be $\varepsilon>0$. By Hale $([8])$ we have that $F(\varphi, \phi)$ is locally Lipschitz with constat $\lambda$ and $(\varphi, \phi) \in X$. Therefore, if we take $x, y \in X$ we have

$$
\begin{aligned}
\| S(s, s-\tau) x- & S(s, s-\tau) y \| \leqslant \\
& \leqslant K e^{-\alpha \tau}\|x-y\|+\int_{s}^{s-\tau} K_{2}\|S(\theta, s) x-S(\theta, s) y\| e^{-\alpha \theta} d \theta,
\end{aligned}
$$

and using the Gronwall lemma and taking $\|x-y\|<\left(K e^{-\alpha \tau+\frac{K \lambda}{\alpha}}\right)^{-1}$ we can conclude that $\|S(s, s-\tau) x-S(s, s-\tau) y\|<\varepsilon$. So for each $t \in \mathbb{R}$ and $\tau>0,\{S(s, s-\tau): s \leqslant t\}$, is equicontinuous. Therefore we can apply Theorem 2.12 .

\section{Pullback asymptotically compact processes}

In this section we prove the theorems associated to the extension of Theorem 1.5 (Theorems 2.3 and 2.5 ) and to the verification of its hypothesis (Theorem 2.8). We also give an application of Theorem 2.5 .

We start the section with some basic results and with the proof of Theorem 2.2 .

Lemma 3.1. Let $\{S(t, s): t \geqslant s\}$ be an evolution process in a metric space $X$. If $B \subset X$, then $S(t, s) \omega(B, s) \subset \omega(B, t)$. If $B$ is such that $\omega(B, s)$ is compact and pullback attracts $B$ at time $s$, then $S(t, s) \omega(B, s)=\omega(B, t)$. Furthermore, if $\omega(B, t)$ pullback attracts $C$ at time $t$ and $C$ is a connected set which contains $\bigcup_{s \leqslant t} \omega(B, s)$, then $\omega(B, t)$ is connected.

Proof: If $\omega(B, t)=\emptyset$, there is nothing to show. If $\omega(B, s) \neq \emptyset$, from the continuity of $S(t, s)$ and from (2.1) one immediately sees that $S(t, s) \omega(B, s) \subset \omega(B, t)$.

It remains to show that, if $\omega(B, s)$ is compact and pullback attracts $B$, then $\omega(B, t) \subset$ $S(t, s) \omega(B, s)$. For $x \in \omega(B, t)$, there are sequences $\sigma_{k} \rightarrow-\infty, \sigma_{k} \leqslant t$ and $x_{k} \in B$ such that $S\left(t, \sigma_{k}\right) x_{k} \stackrel{k \rightarrow \infty}{\longrightarrow} x$. Since $\sigma_{k} \rightarrow-\infty$ we have that there exists $k_{0} \in \mathbb{N}$ such that $\sigma_{k} \leqslant s$ for all $k \geqslant k_{0}$. Hence $S(t, s) S\left(s, \sigma_{k}\right) x_{k}=S\left(t, \sigma_{k}\right) x_{k} \rightarrow x$ for $k \geqslant k_{0}$. Since $\omega(B, s)$ is compact and pullback attracts $B$ at time $s$, we have that $\operatorname{dist}\left(S\left(s, \sigma_{k}\right) x_{k}, \omega(B, s)\right) \stackrel{k \rightarrow \infty}{\longrightarrow} 0$. It is then easy to see that $\left\{S\left(s, \sigma_{k}\right) x_{k}\right\}_{k \in \mathbb{N}}$ has a convergent subsequence (which we again denote by $\left.S\left(s, \sigma_{k}\right) x_{k}\right)$ to some $y \in \omega(B, s)$. It follows from the continuity of $S(t, s)$ that $S(t, s) y=x$. Hence $\omega(B, t)=S(t, s) \omega(B, s)$.

Now we prove the assertion about the connectedness of $\omega(B, t)$. Suppose that $\omega(B, t)$ is disconnected, then $\omega(B, t)$ is a disjoint union of two compact sets (hence separated by a positive distance), but $\omega(B, t)$ pullback attracts $C$ and this is in contradiction with the fact that $S(t, s) C$ is connected and contains $\omega(B, t) \cdot \square$

Lemma 3.2. Let $\{S(t, s): t \geqslant s\}$ be an evolution process in a metric space $X$. If $B$ is a nonempty subset of $X$ such that $\overline{\bigcup_{s \leqslant s_{0}} S(t, s) B}$ is compact, for some $s_{0} \in \mathbb{R}, s_{0} \leqslant t$, then $\omega(B, t)$ is nonempty, compact, invariant and $\omega(B, t)$ pullback attracts $B$ at time $t$. 
Proof: Since $\overline{\bigcup_{s \leqslant \sigma} S(t, s) B}$ is nonempty and compact for each $\sigma \leqslant s_{0}$, we have that $\omega(B, t)$ is nonempty and compact.

Let us show that $\omega(B, t)$ pullback attracts $B$ at time $t$. Suppose not, then there exists $\epsilon>0$ and sequences $\left\{x_{k}\right\}_{k \in \mathbb{N}}$ in $B,\left\{\sigma_{k}\right\}_{k \in \mathbb{N}}$ in $\mathbb{R}$ with $\sigma_{k} \leqslant t, \sigma_{k} \stackrel{k \rightarrow \infty}{\longrightarrow}-\infty$, such that $\operatorname{dist}\left(S\left(t, \sigma_{k}\right) x_{k}, \omega(B, t)\right)>\epsilon$ for all $k \in \mathbb{N}$. Since $\overline{\bigcup_{s \leqslant s_{0}} S(t, s) B}$ is compact and $\left\{S\left(t, \sigma_{k}\right) x_{k}, k \geqslant k_{0}\right\} \subset \overline{\bigcup_{s \leqslant s_{0}} S(t, s) B}$ for some $k_{0} \in \mathbb{N},\left\{S\left(t, \sigma_{k}\right) x_{k}: k \in \mathbb{N}\right\}$ has a convergent subsequence to some $y \in \omega(B, t)$. This leads to a contradiction and shows that $\omega(B, t)$ pullback attracts $B$ at time $t$.

From Lemma 3.1, $\omega(B, t)$ is invariant and the proof is complete. $\square$

Proof of Theorem 2.2: If $\{S(t, s): t \geq s\}$ has a pullback attractor $\{\mathcal{A}(t): t \in \mathbb{R}\}$, each $\mathcal{A}(t)$ is compact and pullback attracts bounded subsets of $X$.

To prove the converse we proceed as follows. First note that, as an immediate consequence of (2.1) we have that $\omega(B, t) \subset K(t)$, for all $B \subset Z$ bounded and all $t \in \mathbb{R}$. Moreover, we also have that $\omega(B, t)$ attracts $B$. Indeed, if not there exists $\varepsilon>0$, a sequence $\left\{s_{n}\right\}_{n \in \mathbb{N}}$ of real numbers with $s_{n} \rightarrow-\infty$ and a sequence $\left\{x_{n}\right\}_{n \in \mathbb{N}}$ in $B$ such that $\operatorname{dist}\left(S\left(t, s_{n}\right) x_{n}, \omega(B, t)\right)>\varepsilon$ for all $n \in \mathbb{N}$. Since $K(t)$ pullback attracts $B$ we have that $\operatorname{dist}\left(S\left(t, s_{n}\right) x_{n}, K(t)\right) \stackrel{n \rightarrow \infty}{\longrightarrow} 0$. Consequently, $\left\{S\left(t, s_{n}\right) x_{n}\right\}_{n \in \mathbb{N}}$ has a convergent subsequence to some $x_{0} \in K(t)$. Hence $x_{0} \in \omega(B, t)$ which leads to a contradiction.

Note that we are now in the hypotheses of Lemma 3.1, which imply the invariance of $\omega(B, t)$.

Thus, defining $\mathcal{A}(t)$ by $(2.2), \mathcal{A}(t)$ is clearly compact and pullback attracts bounded subsets of $X$. The invariance of $\mathcal{A}(t)$ holds from the invariance of each set $\omega(B, t)$. Indeed, given $x_{0} \in \mathcal{A}(s)$, there exists $x_{n} \in \omega\left(B_{n}, s\right)$ with $x_{n} \rightarrow x_{0}$ as $n \rightarrow+\infty$. Then, $S(t, s) x_{n}=y_{n} \in$ $\omega(B, t)$, and by the continuity of the process $S(t, s)$ we have that $S(t, s) x_{n}=y_{n} \rightarrow S(t, s) x_{0}$, which implies that $S(t, s) x_{0} \in \mathcal{A}(t)$. Now, take $y_{0} \in \mathcal{A}(t)$. Then, there exists $y_{n} \in \omega\left(B_{n}, t\right)$ with $y_{n} \rightarrow y_{0}$ as $n \rightarrow+\infty$. But then, again by the invariance of the family $\omega\left(B_{n}, t\right)$, there exists $x_{n} \in \omega\left(B_{n}, s\right)$ with $S(t, s) x_{n}=y_{n}$. But each $S(t, s) x_{n} \in S(t, s) \omega\left(B_{n}, s\right) \subset S(t, s) \mathcal{A}(s)$. As this last set is compact and does not depend on $n$, we get that $\lim _{n \rightarrow+\infty} S(t, s) x_{n}=y_{0} \in$ $S(t, s) \mathcal{A}(s)$.

Given that $\hat{\mathcal{A}}(t)$ is bounded and pullback attracts bounded sets at $t$ we have that $\omega(B, t) \subseteq$ $\hat{\mathcal{A}}(t)$, for all bounded subset $B$ of $X$. Hence $\mathcal{A}(t) \subseteq \hat{\mathcal{A}}(t) \cdot \square$

Now we prove Theorems 2.3 and 2.5 . To that end we need the following auxiliary result

Lemma 3.3. If $\{S(t, s): t \geqslant s\}$ is a pullback asymptotically compact evolution process and $B$ is a nonempty bounded subset of $X$ such that $\overline{\bigcup_{\tau \leqslant s_{0}} S(t, \tau) B}$ is bounded, for some $s_{0} \in(-\infty, t]$, then $\omega(B, t)$ is nonempty, compact, invariant and pullback attracts $B$ at time $t$. 
Proof: First, note that, for any sequences $\left\{x_{k}: k \in \mathbb{N}\right\}$ in $B$ and $\left\{s_{k}: k \in \mathbb{N}\right\}$ in $\left(-\infty, s_{0}\right]$, $s_{k} \stackrel{k \rightarrow \infty}{\longrightarrow}-\infty$, we have that $\left\{S\left(t, s_{k}\right) x_{k}: k \in \mathbb{N}\right\}$ is bounded. It follows from the fact that $\{S(t, s): t \geqslant s\}$ is pullback asymptotically compact that there exists $y \in X$ and subsequence of $\left\{S\left(t, s_{k}\right) x_{k}: k \in \mathbb{N}\right\}$ (which we denote the same) such that $y=\lim _{k \rightarrow \infty} S\left(t, s_{k}\right) x_{k}$. It follows that $y \in \omega(B, t)$ and $\omega(B, t)$ is nonempty.

Now, given a sequence $\left\{y_{k}: k \in \mathbb{N}\right\}$ in $\omega(B, t)$, there are $x_{k} \in B$ and $s_{k} \in\left(-\infty, s_{0}\right]$, $s_{k} \leqslant-k$, such that $\mathrm{d}\left(S\left(t, s_{k}\right) x_{k}, y_{k}\right) \leqslant \frac{1}{k}$. Since $\left\{S\left(t, s_{k}\right) x_{k}: k \in \mathbb{N}\right\}$ has a convergent subsequence, it follows that $\left\{y_{k}: k \in \mathbb{N}\right\}$ has a convergent subsequence and $\omega(B, t)$ is compact.

We now show that $\omega(B, t)$ pullback attracts $B$. If not, there exists $\epsilon>0$ and sequences $x_{k} \in$ $B$ and $s_{k} \stackrel{k \rightarrow \infty}{\longrightarrow}-\infty$, such that $\operatorname{dist}\left(S\left(t, s_{k}\right) x_{k}, \omega(B, t)\right)>\epsilon$. From the pullback asymptotic compactness, there is a $y \in X$ and a subsequence of $\left\{S\left(t, s_{k}\right) x_{k}: k \in \mathbb{N}\right\}$ (which we denote the same) such that $S\left(t, s_{k}\right) x_{k} \stackrel{k \rightarrow \infty}{\longrightarrow} y$. Clearly $y \in \omega(B, t)$ and that leads to a contradiction. It follows that $\omega(B, t)$ attracts $B$.

The invariance of $\omega(B, t)$ now follows from Lemma 3.1 and the result is proved.

Proof of Theorem 2.3: Observe that we are in the hypotheses of Lemma 3.3, so that, given a bounded subset $B$ of $X, \omega(B, t)$ is nonempty, compact, invariant and pullback attracts $B$ at time $t$. Hence, if $\mathcal{A}(t)$ is defined by $(2.2),\{\mathcal{A}(t): t \in \mathbb{R}\}$ is closed, invariant and pullback attracts bounded subsets of $X$. If $B(t)$ is closed and pullback attracts bounded sets at time $t$, it is clear that $\omega(B, t) \subset B(t)$ for each bounded subset $B$ of $X$ and consequently $\mathcal{A}(t) \subset B(t)$. This completes the proof. $\square$

Proof of Theorem 2.5: If $\mathcal{A}(t)$ is given by (2.2), it follows from Theorem 2.3 that $\mathcal{A}(t)$ is closed, invariant, pullback attracts bounded subsets of $X$ at time $t$ and that $\mathcal{A}(t)$ is minimal among the closed sets that pullback attract bounded subsets of $X$ at time $t$. From the fact that $\{S(t, s): t \geqslant s \in \mathbb{R}\}$ is pullback strongly bounded dissipative, there exists a bounded subset $B(t)$ of $X$ that pullback absorbs bounded subsets of $X$ at time $\tau$, for each $\tau \leqslant t$. Since $\omega(B(t), t)$ pullback attracts $B(t)$ at time $t$ (considered as a fixed bounded subset of $X$ ), it pullback attracts every bounded subset of $X$ at time $t$. Indeed, it is enough to prove that, given a bounded subset $D$ of $X, \omega(D, t) \subset \omega(B(t), t)$. If $x_{0} \in \omega(D, t)$, there are sequences $\left\{s_{k}\right\}_{k \in \mathbb{N}}$ in $(-\infty, t]$ with $s_{k} \stackrel{k \rightarrow \infty}{\longrightarrow}-\infty$, and $\left\{x_{k}\right\}_{k \in \mathbb{N}}$ in $D$ such that $S\left(t, s_{k}\right) x_{k} \stackrel{k \rightarrow \infty}{\longrightarrow} x_{0}$. Since $\{S(t, s): t \geqslant s\}$ is pullback strongly bounded dissipative, given a sequence $\left\{\tau_{n}\right\}_{n \in \mathbb{N}}$ with $\tau_{n} \stackrel{n \rightarrow \infty}{\longrightarrow}-\infty$, there exists a sequence $\left\{\sigma_{n}\right\}_{n \in \mathbb{N}}$ with $\sigma_{n} \leqslant \tau_{n}$ such that $S\left(\tau_{n}, s\right) D \subset B(t)$, for all $s \leqslant \sigma_{n}\left(\tau_{n}\right)$. Given that $s_{k} \stackrel{k \rightarrow \infty}{\longrightarrow}-\infty$, for each $\tau_{n}$ there exists $k_{n} \geqslant n$ such that $S\left(\tau_{n}, s_{k_{n}}\right) x_{k_{n}} \in B(t)$. Thus,

$$
S\left(t, s_{k_{n}}\right) x_{k_{n}}=S\left(t, \tau_{n}\right) S\left(\tau_{n}, s_{k_{n}}\right) x_{k_{n}} \in S\left(t, \tau_{n}\right) B(t),
$$


which implies $x_{0} \in \omega(B(t), t)$. This proves that $\mathcal{A}(t) \subset \omega(B(t), t)$ and consequently $\mathcal{A}(t)$ is compact. Since clearly $\omega(B(t), t) \subset \mathcal{A}(t)$ we have that $\mathcal{A}(t)=\omega(B(t), t)$.

Finally, since $\{S(t, s): t \geqslant s\}$ is pullback strongly bounded dissipative, for each bounded subset $D$ of $X, \omega(D, \tau) \subset \overline{B(t)}$, for all $\tau \leqslant t$. In fact, for any $x_{0} \in \omega(D, \tau)$ there is a sequence $\left\{s_{n}\right\}_{n \in \mathbb{N}}$ in $(-\infty, t]$ with $s_{n} \stackrel{n \rightarrow \infty}{\longrightarrow}-\infty$ and $\left\{x_{n}\right\}_{n \in \mathbb{N}}$ in $D$ such that $\lim _{n \rightarrow+\infty} S\left(\tau, s_{n}\right) x_{n}=x_{0}$. Hence, $S\left(\tau, s_{n}\right) x_{n} \in B(t)$ for all suitably large $n$ and so $x_{0} \in \overline{B(t)}$. This implies $A(\tau) \subset \overline{B(t)}$ for all $\tau \leqslant t$ and completes the proof. $\square$

In [8, Lemma 3.2.6] a sufficient condition for a semigroup to be asymptotically compact is given. Next we prove Theorem 2.8 which is its analogue for evolution processes. Let us briefly recall the definition and basic properties of the Kuratowski measure of non-compactness (see [7] for details).

Definition 3.4. Let $X$ be a metric space and $A \subset X$. The Kuratowski measure of noncompactness is defined as:

$$
\alpha(A)=\inf \{\delta>0: \text { A has a finite cover of diameter }<\delta\} .
$$

Lemma 3.5. Let $X$ be a metric space and $\alpha$ the Kuratowski measure:

(1) $\alpha(B)=0 \Leftrightarrow \bar{B}$ is compact;

(2) If $X$ is a Banach space and $B_{1}, B_{2} \subset X \Rightarrow \alpha\left(B_{1}+B_{2}\right) \leqslant \alpha\left(B_{1}\right)+\alpha\left(B_{2}\right)$;

(3) If $B_{1} \subset B_{2} \Rightarrow \alpha\left(B_{1}\right) \leqslant \alpha\left(B_{2}\right)$;

(4) $\alpha\left(B_{1} \cup B_{2}\right) \leqslant \max \left\{\alpha\left(B_{1}\right), \alpha\left(B_{2}\right)\right\}$;

(5) $\alpha(\bar{B})=\alpha(B)$;

(6) $\alpha(k B)=|k| \alpha(B)$ for all $k \in \mathbb{R}$.

Lemma 3.6. Let $X$ be a complete metric space and $\left\{F_{n}\right\}$ a decreasing sequence of nonempty, bounded and closed sets such that $\alpha\left(F_{n}\right) \stackrel{n \rightarrow \infty}{\longrightarrow} 0$. Then $\bigcap_{n \in \mathbb{N}} F_{n}$ is nonempty and compact.

Proof of Theorem 2.8: Let $\left\{x_{n}\right\} \subset B$ with $B \subset X$ bounded and $t_{n}, s_{n} \in \mathbb{R}$ with $t_{n}-s_{n} \rightarrow$ $\infty$. We denote

$$
B_{t}=\bigcup_{\tau \leqslant t} \gamma_{p}(B, \tau),
$$

where $r>0$ is such that $\forall x \in B_{t},\|x\| \leqslant r$ (observe that $B_{t}$ is a bounded set, so there exists $r>0$ such that $\left.B_{t} \subset B(0, r)=B_{r}\right)$. We define the sets

$$
J_{j}=\left\{S\left(t_{n}, s_{n}\right) x_{n}: n \geqslant j\right\} .
$$

For each $x_{n}$ we can write

$$
S\left(t_{n}, s_{n}\right) x_{n}=S\left(t_{n}, \tau_{n}\right) S\left(\tau_{n}, s_{n}\right) x_{n} \subset S\left(t_{n} \tau_{n}\right) B_{r},
$$

with $\tau_{n}=\frac{t_{n}-s_{n}}{2}$. 
EXISTENCE OF PULLBACK ATTRACTORS FOR PULLBACK ASYMPTOTICALLY COMPACT PROCESSES

Let $T_{B}$ be as in Definition 2.9. Then, for all $j \in \mathbb{N}$ such that $t_{j}-\tau_{j} \geqslant T_{B}$ we have

$$
\begin{aligned}
\alpha\left(J_{j}\right) & \leqslant \alpha\left(\left\{T\left(t_{n}, \tau_{n}\right) B_{r}+U\left(t_{n}, \tau_{n}\right) B_{r}: n \geqslant j\right\}\right) \\
& \leqslant \alpha\left(\left\{T\left(t_{n}, \tau_{n}\right) B_{r}: n \geqslant j\right\}\right) \\
& \leqslant k\left(t_{j}-\tau_{j}, r\right) \rightarrow 0 \text { when } j \rightarrow \infty .
\end{aligned}
$$

Since $\alpha\left(J_{1}\right)=\alpha\left(J_{j}\right)$ we conclude that $\left\{S\left(t_{n}, s_{n}\right) x_{n}\right\}$ is a precompact set in $X$.

\section{Pullback Strong Point Dissipativeness}

This section is devoted to the proof of Theorem 2.12. Our main task is to prove that, under certain smoothing properties of the evolution processes, strong pullback point dissipativeness implies strong pullback bounded dissipativeness. The following lemma plays an important rol in that procedure.

Lemma 4.1. Let $\{S(t, s): t \geqslant s\}$ be a pullback strongly point dissipative, pullback asymptotically compact and pullback strongly bounded evolution process. If, for each $t \in \mathbb{R}$ and $\sigma>0$, the family $\{S(\tau, \tau-\sigma): \tau \leqslant t\}$ is equicontinuous at each $x \in X$, then $\{S(t, s): t \geqslant s\}$ is pullback strongly compact dissipative.

Proof: Fix $t \in \mathbb{R}$ and let $B(t)$ be a bounded subset of $Z$ which strongly absorbs points of $X$ at time $t$.

For $\tau \leqslant t$, let $B^{1}(t)=\{x \in X: \mathrm{d}(x, y)<1$ for some $y \in B(t)\}$ and $C(\tau)=\gamma_{p}\left(\overline{B^{1}(t)}, \tau\right)$. Then $C(\tau)$ is a bounded subset of $X$ which strongly absorbs points of $X$ at time $\tau$. Indeed, given $x \in X$ let $\sigma_{x}$ be such that $S(r, s) x \in B(t), s+\sigma_{x} \leqslant r \leqslant \tau \leqslant t$. Then, since $B(t) \subset C(\tau)$, it follows that $C(\tau)$ pullback strongly absorbs points at time $\tau$.

Due to the equicontinuity of the process, if $K$ is a compact subset of $X$ and $x \in K$ there are $\nu_{x} \in \mathbb{N}$ and $\epsilon_{x}>0$ such that $S\left(r, r-\nu_{x}\right) B_{\epsilon_{x}}(x) \subset B^{1}(t)$, for all $r \leqslant \tau$. It follows that $S\left(\tau, r-\nu_{x}\right)\left(B_{\epsilon_{x}}(x)\right) \subset C(\tau)$ for all $r \leqslant \tau$. Since $K$ is compact there is a $p \in \mathbb{N}^{*}$ and $x_{1}, \cdots, x_{p}$ in $K$ such that $K \subset \cup_{i=1}^{p} B_{\epsilon_{x_{i}}}\left(x_{i}\right)$ and for $\sigma_{K}=\max \left\{\sigma_{x_{i}}: 1 \leqslant i \leqslant p\right\}, S\left(\tau, r-\sigma_{K}\right) K \subset C(\tau)$ for all $r \leqslant \tau$. Then, from the fact that $\{S(t, s): s \leqslant t\}$ is pullback strongly bounded, it follows that $\bigcup_{\tau \leqslant t} C(\tau)$ is bounded and pullback strongly absorbs compact subsets of $X$ at time $t \cdot \square$

Theorem 4.2. If a process $\{S(t, s): t \geqslant s\}$ is pullback strongly compact dissipative and pullback strongly asymptotically compact, then $\{S(t, s): t \geqslant s\}$ is pullback strongly bounded dissipative.

Proof: From the fact that $S(t, s)$ is pullback strongly compact dissipative there is a $B(t)$ closed and bounded which pullback strongly absorbs compact subsets of $X$ at time $t$. First we prove that, for each bounded subset $D$ of $X, \omega(D, \tau) \subset B(t)$ for each $\tau \leqslant t$. If $y \in \omega(D, \tau)$, there is a sequence $\left\{s_{k}: k \in \mathbb{N}\right\}$ with $s_{k} \leqslant \tau$ and $s_{k} \stackrel{k \rightarrow \infty}{\longrightarrow}-\infty$ and a sequence $\left\{x_{k}\right\}_{k \in \mathbb{N}} \subset D$ 
such that $\operatorname{dist}\left(S\left(\tau, s_{k}\right) x_{k}, \omega(D, \tau)\right) \stackrel{k \rightarrow \infty}{\longrightarrow} 0$. Taking $\left\{r_{k}: k \in \mathbb{N}\right\}$ with $\tau \geqslant r_{k} \geqslant s_{k}$ and $\min \left\{\tau-r_{k}, r_{k}-s_{k}\right\} \stackrel{k \rightarrow \infty}{\longrightarrow} \infty$ and using the fact that $\{S(t, s): t \geqslant s\}$ is pullback strongly asymptotically compact (taking subsequences if necessary), there is a $z \in X$ such that $z_{k}:=S\left(r_{k}, s_{k}\right) x_{k} \stackrel{k \rightarrow \infty}{\longrightarrow} z$. From the compactness of the set $K=\left\{z_{k}: k \in \mathbb{N}\right\} \cup\{z\}$, there is a $\sigma_{K} \in \mathbb{N}$ such that $S\left(\tau, r_{k}\right) K \subset B(t)$ whenever $\tau-r_{k} \geqslant \sigma_{K}$. Thus, for all suitably large $k$,

$$
S\left(\tau, s_{k}\right) x_{k}=S\left(\tau, r_{k}\right) S\left(r_{k}, s_{k}\right) x_{k} \subset S\left(\tau, r_{k}\right) K \subset B(t) .
$$

This completes the proof that $\omega(D, \tau) \subset B(t)$ for each $\tau \leqslant t$.

Since $\omega(D, \tau)$ pullback attracts $D$ at time $\tau$, it follows that $B(t)$ pullback attracts bounded subsets of $X$ at time $\tau$ for each $\tau \leqslant t$; that is, $\{S(t, s): t \geqslant s\}$ is pullback strongly bounded dissipative. $\square$

As an immediate consequence of Theorem 2.5 we have that

Theorem 4.3. If a process $\{S(t, s): t \geqslant s\}$ is pullback strongly compact dissipative and pullback strongly asymptotically compact, then $\{S(t, s): t \geqslant s\}$ has a pullback attractor $\{\mathcal{A}(t): t \in \mathbb{R}\}$ with the property that $\bigcup_{s \leqslant t} \mathcal{A}(s)$ is bounded for each $t \in \mathbb{R}$.

The proof of Theorem 2.12 is now a direct application of Lemma 4.1 and Theorem 4.3 .

Final Remark Although, for simplicity, the results given in this work concern the pullback attractor of bounded sets, they can be generalized in such way that other classes of sets can be attracted. In that case, the introduced definitions of dissipation and asymptotic compactness should be changed accordingly to cope with these other classes (see [2] for more details).

Acknowledgement We would like to thank the anonymous referee for interesting comments and suggestions.

\section{REFERENCES}

[1] A. B. Babin, M.I. Vishik, Attractors of evolution equations. North Holland (1992).

[2] T. Caraballo, G. Lukaszewicz, J. Real, Pullback attractors for asymptotically compact non-autonomous dynamical systems. Nonlinear Analysis 64 (2006), 484-498 .

[3] D. Cheban, P. E. Kloeden, B. Schmalfuß, The relationship between pullback, forwards and global attractors of nonautonomous dynamical systems, Nonlinear Dyn. Syst. Theory 2 125-144 (2002).

[4] D. N. Cheban, Global Attractors of Non-Autonomous Dissipative Dynamical Systems. World Scientific Publishing Co. Pte. Ltd (2004).

[5] D. N. Cheban, P.E. Kloeden, B. Schmalfuss, The relationship between pullback, forwards and global attractors of nonautonomous dynamical systems. Nonlinear Dynamics \& Systems Theory, in press.

[6] V. V. Chepyzhov, M.I. Vishik, Attractors for Equations of Mathematical Physics. Colloquium Publications, Vol. 49. America Mathematical Society (2002). 
[7] K. Deimling, Nonlinear Functional Analysis. Springer-Verlag (1985).

[8] J. K. Hale, Asymtotic Behavior of Dissipative System. American Mathematical Society (1989).

[9] P. E. Kloeden, Pullback attractors of nonautonomuos semidynamical system. Stochastics and Dynamics, Vol. 3, No. 1 (2003), 101-112.

[10] O. Ladyzhenskaya, Attractors for semigroups and evolution equations. Lezioni Lincee. [Lincei Lectures] Cambridge University Press, Cambridge, 1991.

[11] Q. Ma, S. Wang, Ch. Zhong, Necessary and sufficient Conditions for the Existence of Global Attractors for semigroups and Applications. Indiana University Mathematics Journal, Vol. 51, No. 6 (2000), 15411559.

[12] G. Raugel, Global Attractor in Partial Differential Equations, preprint.

[13] J. C. Robinson, Infinite-Dimensional Dynamical System. An introduction to dissipative parabolic PDEs and the theory of global attractors. Cambridge Text in Applied Mathematics (2001).

[14] B. Schmalfuß, Attractors for the nonautonomous dynamical systems. International Conference of Diffential Equations, Vol. 1, 2 (1999).

[15] G. R. Sell, Y. You, Dynamics of evolutionary equations. Applied Mathematical Sciences, 143. SpringerVerlag (2002).

[16] G. R. Sell, Nonautonomous differential equations and dynamical systems. Trans. Amer. Math. Soc. no. 127 (1967), 241-283 .

[17] R. Temam, Infinite-Dimensional Dynamical System in Mechanics and Physics. Springer-Verlag (1988).

(T. Caraballo) Departamento de Ecuaciones Diferenciales y Análisis Numérico, Universidad de Sevilla, Apdo. de Correos 1160, 41080-Sevilla Spain

(A. N. Carvalho) Instituto de Ciências Matemáticas e de Computaçao, Universidade de São Paulo-Campus de São Carlos, Caixa Postal 668, 13560-970 São Carlos SP, Brazil

(J. A. Langa) Departamento de Ecuaciones Diferenciales y Análisis Numérico, Universidad de Sevilla, Apdo. de Correos 1160, 41080-Sevilla Spain

(F. Rivero) Departamento de Ecuaciones Diferenciales y Análisis Numérico, Universidad de Sevilla, Apdo. de Correos 1160, 41080-Sevilla Spain 